\title{
Magnetic Attraction of Gaze: Further Evidence of Hemispheric Imbalance in Schizophrenia?
}

\author{
S. B. UNNITHAN, A. S. DAVID and J. C. CUTTING \\ Maudsley Hospital, Denmark Hill, London SE5 8AZ, UK \\ Correspondence: Dr S. B. Unnithan at above address.
}

\begin{abstract}
A case is reported of a young man with a clinical diagnosis of schizophrenia and unusual oculo-motor abnormalities. The relationship between oculo-motor dysfunction and hemispheric balance in schizophrenia is discussed.
\end{abstract}

\section{Introduction}

Disordered eye movement in schizophrenia has been recognized since the turn of this century (Diefendorf and Dodge, 1908). Several abnormalities have been described involving saccades, smooth pursuit and conjugate lateral eye movements (Holzman et al., 1976; Iacono, 1988).

We now describe a patient with magnetic attraction of gaze, an abnormality previously reported in patients with focal right hemisphere insults (De Renzi, 1988).

In view of the increasing evidence that schizophrenia may have an underlying cerebral dysfunction in the form of hemisphere imbalance (Cutting, 1990) the occurrence of the same phenomenon in schizophrenia is of some interest.

\section{Case Report}

A 28-year-old white male was admitted to the Bethlem Royal Hospital from another psychiatric hospital for a second opinion on diagnosis and management.

His mother, aged 52, suffered from post-partum depression after the birth of her youngest child, and was treated with antidepressants. His father, aged 52, was an office manager with no history of psychiatric or physical illness. The patient had two siblings, a younger brother and sister, neither of whom had suffered from psychiatric illness.

The patient's early personal history included forceps delivery following a long labour but there were no major developmental or childhood problems. He enjoyed school and had many friends until the age of 16 , when he was noted to have become increasingly withdrawn and to have difficulty concentrating, although he still gained 6 CSEs and 2 "O" level examina$0953-4180 / 91 / 040063+04 \$ 3.50 / 0 \quad$ (C) 1991 CNS (Clinical Neuroscience) Publishers 
tions. He then worked in several clerical jobs but he was increasingly impaired by poor concentration.

More frequent episodes of bizarre behaviour and a deterioration in his social interaction were noted by his parents from the ages of 18-25. $\mathrm{He}$ would burn all his clothes, was increasingly socially withdrawn, lacked personal hygiene and adopted an unusual way of walking which involved him turning round every few yards to look behind him.

A diagnosis of schizophrenia was made at the age of 25 . This was based on the observations of his parents who had noted a general deterioration in his personality and level of functioning, and our own observations on the ward of the negative symptoms of schizophrenia, such as apathy, poverty of speech, affective flattening and inexplicable behaviour.

In July 1988 his parents noted that he had started a "fixing" behaviour, fixing his gaze and attention on real objects. This resulted in him opening doors up to twenty times once he had "fixed" his eye on an object inside a room. He was also noted to become increasingly distressed if prevented from "fixing". Admission to his local hospital in September 1988 was precipitated by an episode of aggressive behaviour on attempting to prevent him from "fixing".

Following transfer to our unit his main complaint was of getting "fixed" on items such as light switches, posters, radiator taps, always on the right side of his field of vision. He would then have to look at this object many times until he "got used to them" and the "fixing" then ceased. He described feelings of anxiety if prevented from completion of "fixing" and a sense of relief on completion.

Mental state examination revealed a dishevelled young man, with a flat affect and little emotional reactivity. He described feeling low but not suicidal. His content of speech was normal with no abnormal ideas expressed, and he denied any other abnormal experiences or compulsions.

Physical examination was normal. Neuro-ophthalmological examination showed no evidence of hemianopia, confirmed by perimetry.

Psychological testing revealed that lateralized tachistoscopic $(80 \mathrm{~ms})$ testing of shape, colour and word recognition was normal, apart from some difficulty naming two syllable words in the left visual field (eight out of 12 correct). Matching of these stimuli when two were presented, each 3 degrees on either side of fixation was performed with around $90 \%$ accuracy with no evidence of neglect or extinction. An EEG and CT scan were normal.

Prior to the transfer to the Bethlem Royal Hospital, trifluoperazine orally, fluphenazine decanoate injections and sulpiride orally had been tried but had not been continued due to the patient's non-compliance. During his admission at the Bethlem Royal Hospital his drug treatment was changed to thioridazine $200 \mathrm{mg}$ qds orally with which there was an improvement in his mood and motivation. The frequency of this "fixing" behaviour reduced and he was able to be discharged home.

Since discharge he has attended his local hospital and has shown no evidence of deterioration in the level of "fixing" behaviour, although his general mental state and in particular his lack of activity has remained static. 


\section{Discussion}

This case demonstrates an episodic abnormality of gaze. Eye movements said to be under hemispheric influence include: smooth pursuit eye movements, saccadic eye movements and conjugate lateral eye movements (Lipton et al., 1980). Pursuit movements hold a moving image, steady on the fovea, an example being the slow phase of vestibular nystagmus. Saccadic eye movements are seen where the sudden appearance of a visual stimulus, attracts a subject's attention, requiring a rapid displacement of the eyes; an example of a saccadic eye movement is seen in the quick phase of nystagmus. Conjugate lateral eye movements are described as the movement of the eyes and head in a direction contralateral to the cerebral hemisphere which is activated. A strong association between schizophrenia and smooth pursuit eye movement disruption has been noted in $86 \%$ of chronic schizophrenics and $44 \%$ of first degree relatives of schizophrenics (Holzman et al., 1974). This, plus the fact that early reports occurred prior to the introduction of neuroleptics implies that these eye movement disruptions cannot be attributed to neuroleptic drug effects.

Interpretation of these eye movement disorders has focused on the importance of general non-specific abnormalities of attention. Findings reveal however, that the disorder cannot be linked to simple inattention or lack of motivation (Holzman et al., 1976), and one has to conclude that a dysfunction of control centres is occurring.

Our patient displayed particular features of repeated head and eye movements and direction of attention always towards elements in the right visual field. Eye head synkinesia and episodic unexplained lateral glances have been found to occur more frequently in schizophrenia (Kolada and Pitman, 1983; Stevens, 1978).

An almost identical "magnetic attraction of gaze" or "peeking" had been described by De Renzi as occurring in patients with brain damage. Of 76 consecutive patients with unilateral hemisphere disease, 17 were found to show evidence of "peeking". Thirteen of these had right brain damage (De Renzi, 1988). There was no correlation with hemianopia. Other investigations of the significance of head turning when tested as a lateralizing sign have also shown that head turning is as likely to be due to ipsilateral hemispheric lesions as contralateral (Ochs et al., 1984). We therefore propose that a similar disorder of function in the right hemisphere may be responsible for our patient's symptoms. Posner et al. (1984) provide evidence that damage to the parietal lobe, particularly on the right, produces a specific deficit in the disengagement of attention from its current focus, as well as in the engagement of a new target.

Evidence for the involvement of the left hemisphere in schizophrenia had been well documented, early work noting the association between leftsided temporal lobe epilepsy and schizophrenic type psychoses (Flor-Henry, 1969). Motoric laterality in schizophrenia has also suggested left hemisphere abnormalities (Gurr, 1977).

Recent evidence, however, has accumulated which indicates right hemisphere dysfunction may be present in schizophrenia as seen by abnormalities 
in visuo-spatial processing (Schweitzer, 1982). Although original theories regarding the control centres of ocular motion suggested the frontal eye field and occipital eye field were of primary importance, action-time experiments have suggested that programming oculomotor tasks occurs predominantly in the right hemisphere (Sava et el., 1988).

Neurobiological measures of hemispheric involvement show no clear evidence of lateralization, but conclusions drawn from neuroradiological studies are that the right rather than the left hemisphere show abnormalities (Gur et al., 1985) in schizophrenia.

The symptom cluster reported here when taken in conjunction with previous findings (De Renzi, 1988) lend support to the existence of hemispheric imbalance in schizophrenia.

\section{References}

Cutting, J. G. (1990). Direct examination of hemisphere imbalance in schizophrenia. In "The Right Cerebral Hemisphere and Psychiatric Disorders" (Ed. J. C. Cutting). Oxford University Press, Oxford, pp. 328-345.

De Renzi, E. (1988). Oculomotor disturbances in hemispheric disease. In "Neurology of Eye Movements" (Eds C. W. Johnston and F. J. Pirozzolo). Lawrence Erlbaum Associates, New Jersey, pp. 177-199.

Diefendorf, A. R. and Dodge, R. (1908). An experimental study of the ocular reactions of the insane from photographic records. Brain, 31, 451-489.

Flor-Henry, P. (1969). Psychoses and temporal lobe epilepsy; a controlled investigation. Epilepsia, 10, 363-395.

Gur, R. E. (1977). Motoric laterality imbalance in schizophrenia. Archives of General Psychiatry, 34, 33-37.

Gur, R. E., Gur, R. C., Skolnick, B. E., Caroff, S., Obrist, W. D., Resnick, S., and Reivich, M. (1985). Brain function in psychiatric disorders III. Regional cerebral blood flow in unmedicated schizophrenics. Archives of General Psychiatry, 42, 4, 329-334.

Holzman, P. S., Proctor, L. R., Levy, D. L., Yasillon, N. J., Meltzer, H. Y. and Hurt, S. W. (1974). Eye tracking dysfunctions in schizophrenic patients and their relatives. Archives of General Psychiatry, 231, 143-151.

Holzman, P.S., Levy, D. L. and Proctor, L. R. (1976). Smooth pursuit eye movements, attention and schizophrenia. Archives of General Psychiatry, 33, 1415 -1420.

Iacono, W. G. (1988). Eye movement abnormalities in schizophrenia and affective disorders. In "Neuropsychology of Eye Movements" (Eds C. W. Johnston and F. J. Pirozzolo). Lawrence Erlbaum Associates, New Jersey, pp. 115-145.

Kolada, S. J. and Pitman, R. K. (1983). Eye head synkinesia in schizophrenic adults during a repetitive visual search task. Biological Psychiatry, 18, 6, 675-684.

Lipton, R. B., Levin, S. and Holzman, P.S. (1980). Horizontal and vertical pursuit eye movements, the oculocephalic reflex and the functional psychoses. Psychiatry Research, 3, 193-203.

Ochs, R., Gloor, P., Quesney, F., Ives, J. and Olivier, A. (1984). Does head turning during a seizure have a lateralising or localising significance? Neurology, 34, 884-890.

Posner, M. I., Walker, J. A., Friedrich, F.J. and Rafal, R. D. (1984). Effects of parietal injury on covert orienting of attention. Fournal of Neuroscience, 4, 7, 1863-1874.

Sava, D., Liotti, M. and Rizzolatti, G. (1988). Right hemisphere superiority for programming oculomotion: evidence from simple reaction time experiments. Neuropsychologia, 20, $2,201-211$

Schweitzer, R. L. (1982). Evidence of right cerebral hemisphere dysfunction in schizophrenic patients with left hemisphere overactivation. Biological Psychiatry, 17, 6, 655-673.

Stevens, J. F. (1978). Eye blink and schizophrenia. Psychosis or tardive dyskinesia? American Journal of Psychiatry, 135, 223-226. 


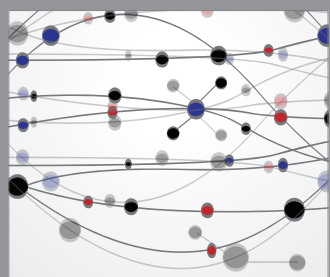

The Scientific World Journal
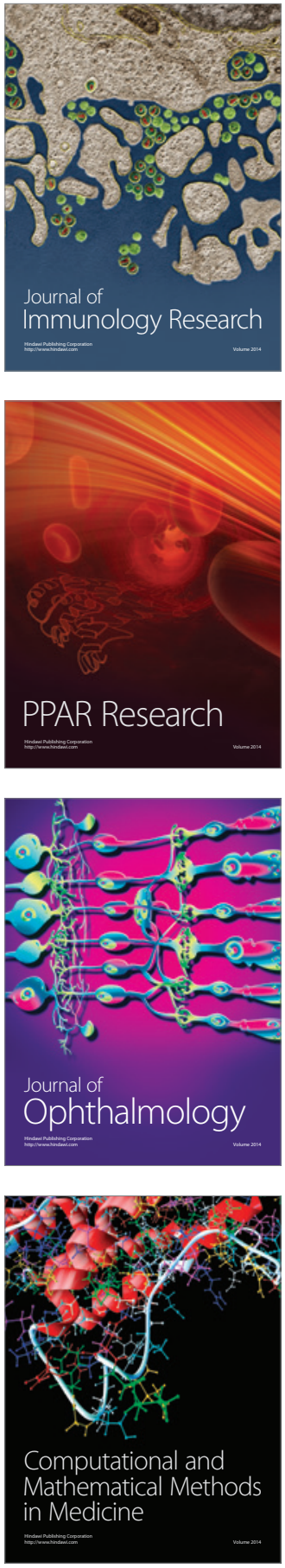

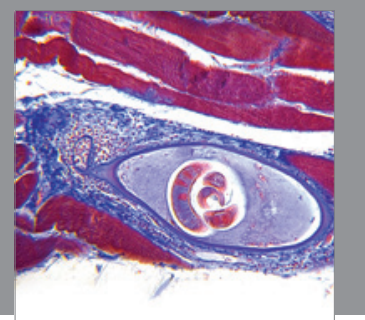

Gastroenterology

Research and Practice
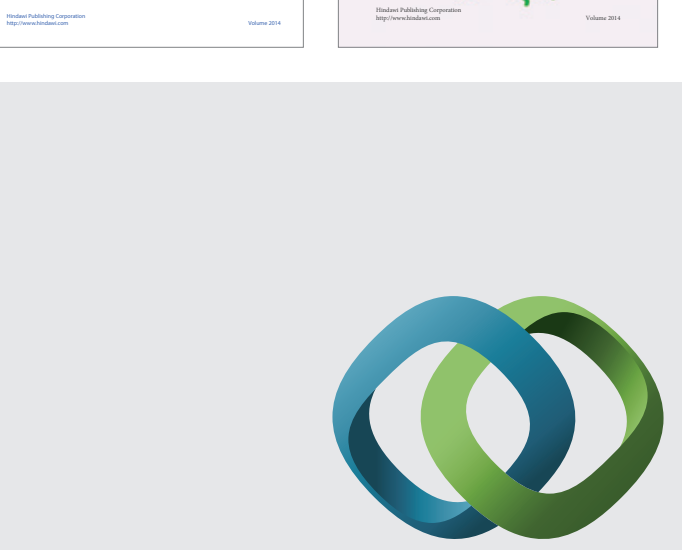

\section{Hindawi}

Submit your manuscripts at

http://www.hindawi.com
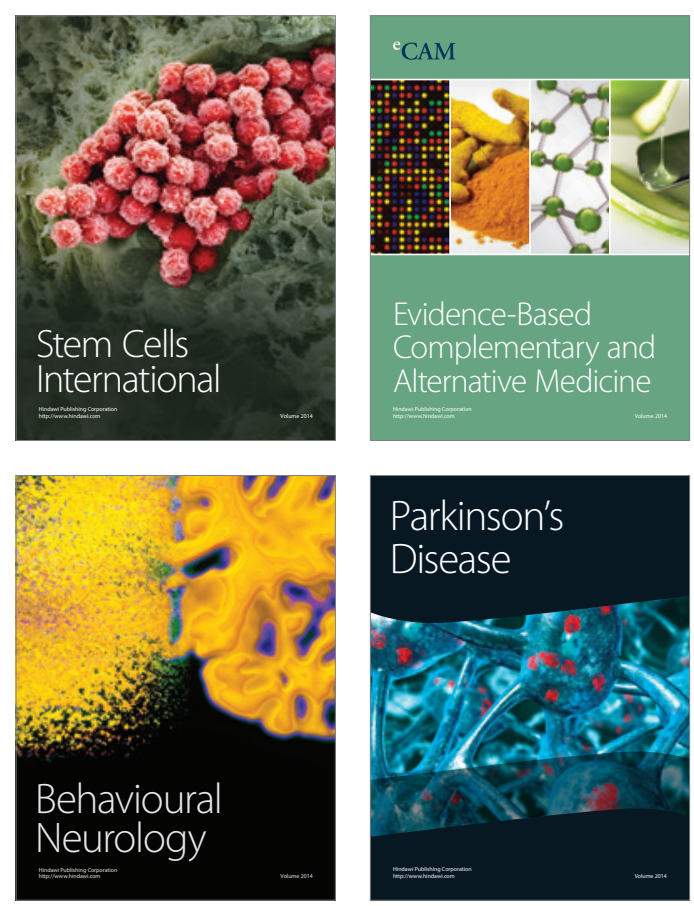

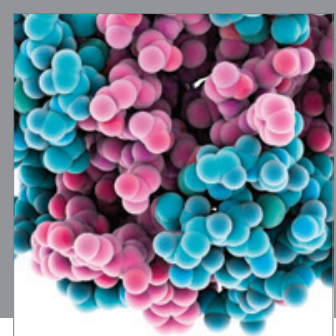

Journal of
Diabetes Research

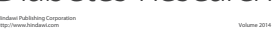

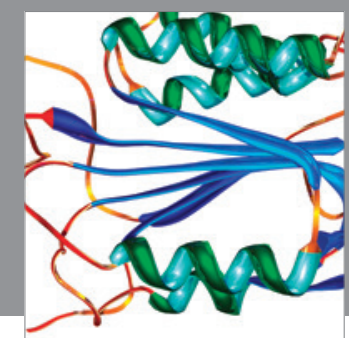

Disease Markers
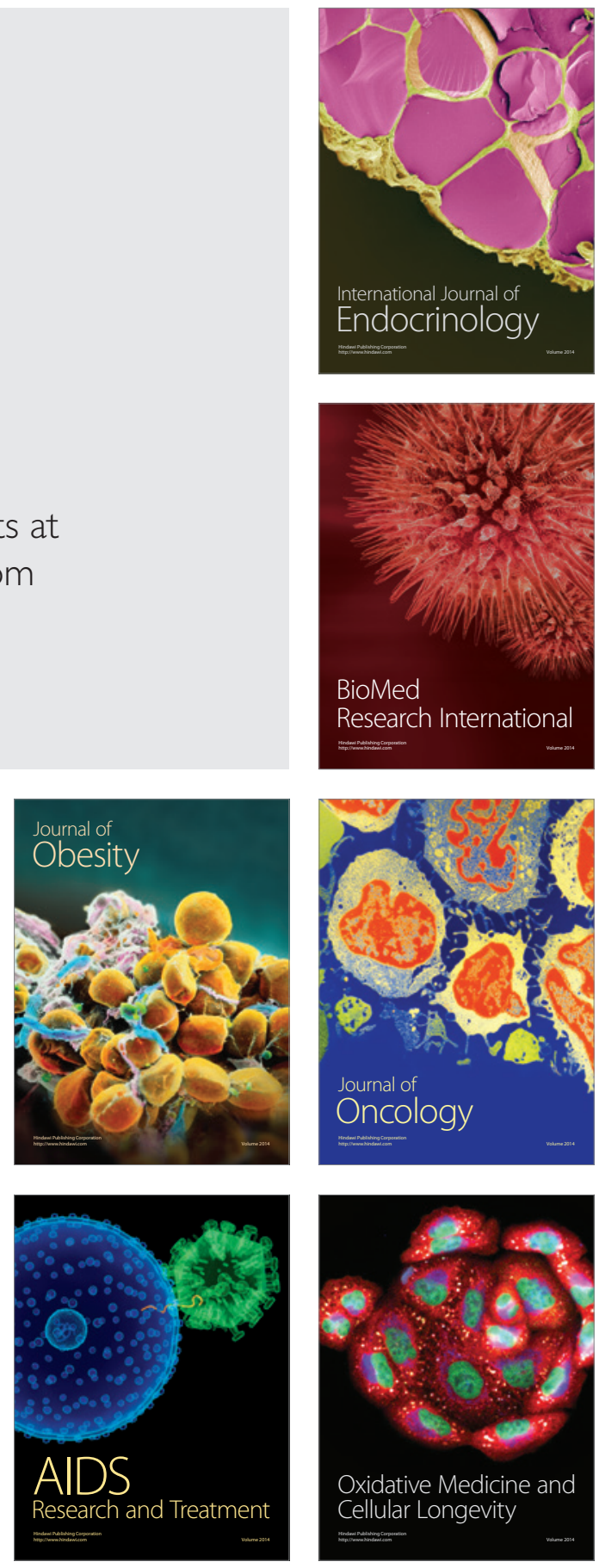